\title{
ACREDITACIÓN Y DESARROLLO DE CAPACIDADES ORGANIZACIONALES EN LAS UNIVERSIDADES CHILENAS ${ }^{1}$
}

\author{
Enrique Fernández Darraz², Claudio Ramos Zincke ${ }^{3}$
}

\begin{abstract}
RESUMEN
Este artículo investiga la relación, en las universidades chilenas, entre los logros en los procesos de acreditación y el desarrollo de capacidades organizacionales. Se utilizaron dos procedimientos de análisis. Primero, una regresión, a partir de un conjunto de variables independientes (año de inicio de la actividad de acreditación en programas e institucional y resultados obtenidos) y dependientes (logros vigentes en la acreditación institucional y de pregrado, y un índice agregado de desarrollo académico institucional). Este análisis fue complementado con otro estudio, que revisó la ubicación de las unidades de calidad en los organigramas, en dos sentidos: uno evolutivo, centrado en su variación entre 2008-2009 y 2019 (37 instituciones), y otro focalizado en el año 2019 (48 instituciones). Se aprecia que todas las universidades analizadas disponen de unidades de calidad y, algunas de ellas, especialmente del sector privado de reciente fundación, las sitúan en las jerarquías más altas. El desarrollo de capacidades organizacionales les ha permitido mejorar sus logros en los procesos de acreditación, generando un círculo virtuoso. Si ello tiene también efectos en la calidad de la enseñanza o en la eficiencia de la gestión, sigue siendo una pregunta abierta, como en la mayoría de la literatura.
\end{abstract}

Conceptos clave: universidades, acreditación, capacidades organizacionales, aseguramiento de la calidad, Chile.

\section{ACCREDITATION AND DEVELOPMENT OF ORGANIZATIONAL CAPABILITIES IN CHILEAN UNIVERSITIES}

\section{ABSTRACT}

This article investigates the relationship between achievements in accreditation processes and the development of organizational capabilities, in Chilean universities. Two methods of $s$ analysis were used. First, a regression, from a set of independent variables (year of initiation of accreditation activity at the program and institutional level, and results obtained) and dependent variables (current achievements in institutional and undergraduate accreditation, and an aggregated index of institutional academic development). This analysis was complemented with another study, which reviewed the location of the quality units in the organization charts, with two perspectives: an evolutionary one, focused on its variation between 2008-2009 and 2019 (37

1 El presente artículo fue escrito en el marco del proyecto Fondecyt Regular 1170477: "La gubernamentalidad del sistema educacional chileno y su dispositivo evaluador: redes de producción, decisiones controversiales y efectos performativos (1968-2019)".

2 Universidad Central, Santiago, Chile. Contacto: enrique.fernandez@ucentral.cl

3 Universidad Alberto Hurtado, Santiago, Chile. Contacto: claudior@uahurtado.cl 
institutions), and another focused on the year 2019 (48 institutions). It can be seen that all the analyzed universities have quality units and some of them, especially the ones recently founded in the private sector, place them in the highest hierarchies. The development of organizational capabilities has allowed them to improve their achievements in the accreditation processes, generating a virtuous circle. Whether this also has effects on the quality of teaching or on the efficiency of management remains an open question, as in most of the literature.

Key words: universities, accreditation, organizational capabilities, quality assurance, Chile. 


\section{Introducción}

Los procesos de acreditación en educación superior comenzaron en Chile a inicios de la década del 2000, luego de la creación, en 1999, de las dos instancias que se harían cargo de dicha actividad: la Comisión Nacional de Acreditación de Pregrado (CNAP) y la Comisión Nacional de Acreditación de Postgrado (CONAP) ${ }^{4}$. Ambas surgieron como comisiones asesoras del Ministerio de Educación, con el cometido específico de realizar procesos experimentales de acreditación, de carácter voluntario, y de preparar un futuro marco legislativo para esta tarea.

Si bien la inquietud por el aseguramiento de la calidad existía desde inicios de los años 90, recién con la constitución de estas comisiones la política pública formalizó dicha preocupación.

La Ley 20.129 (sobre Aseguramiento de la Calidad), promulgada en octubre de 2006, dio estatus legal a dichos procesos. Ella tomó la experiencia acumulada y generó una nueva institución: la Comisión Nacional de Acreditación (CNA), que inició funciones en 2007.

Las críticas a la calidad de la formación terciaria, sin embargo, persistieron. Por esta razón, la recientemente aprobada Ley de Educación Superior ( No 21.091, mayo de 2018) modificó, además de otros aspectos, el sistema de acreditación vigente. Entre los cambios más relevantes están eliminar su carácter voluntario y reemplazar el modelo que evaluaba a las instituciones en relación con el grado de cumplimiento de los propósitos de desarrollo por ellas declarados, por otro basado en criterios y estándares establecidos nacionalmente.

En las casi dos décadas transcurridas desde la creación de ambas comisiones se han llevado a cabo más de 200 acreditaciones institucionales, 2.800 de pregrado y cerca de 1.700 de posgrado, es decir, unos 4.700 procesos. Estas cifras demuestran el alcance que esta preocupación evaluativa ha tenido en la educación superior chilena.

4 Desde inicios de la década de 1990, la Comisión Nacional de Investigación Científica y Tecnológica (Conicyt) realizaba procesos de acreditación de programas de posgrado, con la finalidad de asignar becas. 
Las universidades, como era de esperar, exhiben logros diversos en su acreditación institucional, tanto en lo que se refiere a la cantidad de años otorgada, como al número de áreas evaluadas. Estos disímiles resultados tienen variados orígenes, asociados a las capacidades organizacionales de que disponían al momento de iniciar los procesos, pero también a la evolución que estas experimentaron, precisamente a raíz de las nuevas exigencias de rendición de cuentas, además de otros cambios contextuales.

El presente artículo aborda la relación entre logros históricos en la acreditación institucional y desarrollo de capacidades organizacionales. La proposición central de esta investigación plantea que, a mayor participación en dichos procesos, mayores capacidades y consolidación organizacional de la actividad a través de unidades específicas a cargo de ella y, por lo mismo, mayores logros en la acreditación, generando una relación virtuosa.

Con el objeto chequear dicha conjetura se realizó un análisis de regresión, a partir de un conjunto de variables relacionadas con acreditación institucional y de carreras, tipo de institución y los niveles jerárquicos de las unidades a cargo del aseguramiento de la calidad. Complementariamente se llevó a cabo un análisis de la ubicación y evolución de dichas unidades en la estructura organizacional, con el objetivo de caracterizarlas desde una perspectiva cualitativa.

Por último, se plantean un conjunto de hipótesis teóricas, que buscan abrir caminos a la investigación sobre posibles implicancias organizacionales de los procesos de acreditación de la calidad en universidades.

Anteceden al análisis empírico mencionado dos acápites que tratan la instalación de los procesos de aseguramiento de calidad en el sistema de educación superior chileno y las dificultades encontradas por la literatura para determinar los impactos de dichos procesos en las instituciones de educación superior. 


\section{Trayectoria del Sistema Nacional de Aseguramiento de la Calidad}

La creación, en 1999, de la Comisión de Evaluación de Calidad de Programas de Pregrado de Instituciones Autónomas de Educación Superior, en febrero, y de la Comisión de Evaluación de Calidad de Programas de Postgrado de Universidades Autónomas, en junio, marcó el inicio de la actividad de acreditación impulsada formalmente desde la política pública chilena. No obstante, el aseguramiento de la calidad en este nivel educativo era una preocupación que se había instalado ya a inicios de la década de 1990.

En marzo de 1990, el recién electo presidente Patricio Aylwin nominó una Comisión de Estudios de la Educación Superior con dos cometidos: primero, "estudiar y proponer las modificaciones que estimase necesario introducir en la legislación vigente en el sector de la enseñanza superior" y, segundo, formular las bases para una política de desarrollo del área (Comisión, 1991, p. vii) ${ }^{5}$.

Tanto en el Proyecto de Ley, remitido a la Presidencia de la República en noviembre de 1990, como en la Propuesta de Política para el desarrollo de la Educación Superior en la década de los Nnoventa, entregada en marzo de 1991, la Comisión expresaba su preocupación por el problema de la calidad y proponía medidas para enfrentarlo.

Para ello sugirió la creación de un Consejo Nacional de Educación Superior, que estuviera a cargo de

organizar y administrar el procedimiento de acreditación de los establecimientos de educación superior, el que tiene por objeto la evaluación continua de las instituciones que gocen de plena autonomía y que libremente se acojan a él, con miras a elevar la calidad y la eficiencia del sistema de educación superior. (Comisión, 1991, p. 11)

5 Este grupo de trabajo fue conocido coloquialmente como "Comisión Brunner", dado que fue presidida por el sociólogo José Joaquín Brunner. Existe una segunda "Comisión Brunner", en 1994, con la que suele ser confundida.

6 Esta institución debía reemplazar al Consejo Superior de Educación, que había sido creado por la Ley Orgánica Constitucional de Enseñanza, promulgada en los días finales de la 
Por una combinación de razones, relativas al respeto a la autonomía de las instituciones, a la clara visión de la dificultad política de lograr un consenso legislativo sobre el tema y a la convicción de que la "calidad no puede ser impuesta a los establecimientos mediante medidas administrativas o exigencias burocráticas" (Comisión, 1991, p. 25), la Comisión propuso que la acreditación fuera de carácter voluntario, pero estuviera asociada a determinados beneficios económicos emanados desde la política pública.

El carácter voluntario de los procesos de acreditación salvo en algunas carreras del área de la salud y de pedagogía a partir de $2007^{7}$ - fue uno de los rasgos principales del sistema de aseguramiento de la calidad chileno, ratificado por ley en 2006, y se extendió hasta la reciente aprobación de la nueva Ley de Educación Superior ( $\mathrm{N}^{\circ} 21.091$ ) en mayo de 2018, que estableció su obligatoriedad.

De ese modo, la "Comisión Brunner" se había hecho cargo de una idea que venía desde fines de la década de 1980, en cuanto a que "los mecanismos de regulación vigentes habían sido insuficientes para encauzar la diversificación del sistema y resguardar su desarrollo conforme a exigencias de calidad y estabilidad congruentes con el interés público" (Bernasconi y Rojas, 2003, p. 148).

Las dificultades para lograr acuerdos políticos, sin embargo, impidieron establecer regulaciones sobre éste y otros aspectos de la educación superior. Por este motivo, el proyecto de Ley, ingresado a la Cámara de Diputados en septiembre de 1992 (Mensaje 392-324), que buscaba reformar la Ley Orgánica Constitucional de Enseñanza, no encontró apoyo, siendo archivado en 1997 (Fernández, 2015, p. 188).

dictadura del general Pinochet, en marzo de 1990. El Consejo Nacional de Educación Superior, a propuesta de la Comisión, debía asumir cuatro tareas: la aprobación y supervisión de los proyectos institucionales nuevos, ejercer la función de superintendencia respecto de las instituciones autónomas, organizar y administrar los procesos de acreditación y operar como sistema de información (Comisión, 1991, p. 11).

7 La Ley 20.129 estableció la obligatoriedad de acreditar las carreras conducentes a títulos profesionales de médico cirujano, profesor de educación básica, de educación media, de educación diferencial y educador de párvulos. 
En octubre de 2006 finalmente, luego de más de tres años de discusión parlamentaria, se aprobó la Ley que creó el Sistema Nacional de Aseguramiento de la Calidad ( $N^{\circ}$ 20.129). Esa nueva Ley institucionalizó los procesos de acreditación a partir de la formación de un órgano público de carácter autónomo a cargo de ellos: la Comisión Nacional de Acreditación.

Una de las novedades relevantes de la Ley fue el surgimiento de las agencias de acreditación, que podían constituirse como corporaciones privadas con fines de lucro y que estarían a cargo de acreditar carreras de pregrado y programas de magíster. Las agencias quedaban bajo la supervisión directa de la CNA y serían las propias instituciones educativas quienes las seleccionarían al momento de requerir sus servicios.

Este nuevo sistema de acreditación no logró sortear las críticas que Bernasconi y Rojas (2003) habían identificado ya para la década de 1980 —mencionadas más arriba—, lo que llevó a que, luego de varios años de discusión pública y legislativa, en mayo de 2018 se promulgara una Ley de Educación Superior ( $\left.N^{\circ} 21.091\right)$ que, además de otros muchos aspectos, modificaba el sistema significativamente.

Entre los cambios más relevantes está suprimir el carácter voluntario de los procesos y ceñirlos a un conjunto de criterios y estándares que serían elaborados por la propia Comisión. Es decir, elimina el modelo de evaluación conocido como de "ajustes a propósitos", que consistía en evaluar a las instituciones respecto del grado de consistencia que exhibían entre los objetivos de desarrollo por ellas declarados y su quehacer efectivo en pos de su cumplimiento.

El trabajo iniciado en la década del 2000 por la entonces CNAP, se centró en la acreditación de carreras ${ }^{8}$. No obstante, prontamente la

E El modelo implementado en Chile, tanto en lo que se refería a sus aspectos formales (carácter piloto, procesos voluntarios y régimen de "ciclos" o convocatorias de acreditación) como a su modalidad (autoevaluación interna y visita de pares externos), emuló al Plan Nacional de Evaluación de la Calidad de las Universidades españolas (PNECU, 1993-1994) y al Proyecto Piloto Europeo para la Evaluación de la Calidad de la Enseñanza Superior (1994-1995) (Garreta, 1998; Mairata, 2001). 
Comisión se percató de la necesidad de extender el sistema a un nivel institucional. El éxito de esta iniciativa se debió, entre otros factores, a la incorporación de las universidades más importantes del país, lo que le dio un alto nivel de legitimidad (Zapata, 2004, pp. 144-145).

Los procesos fueron organizados por la CNAP en una modalidad de ciclos de acreditación. El primero tuvo lugar entre mayo de 2003 y abril de 2004, y a él se integraron 14 instituciones: nueve universidades pertenecientes al Consejo de Rectores, tres privadas y dos institutos profesionales. Al siguiente (septiembre de 2003 a octubre de 2004) se agregaron otras 20 y al tercer ciclo, entre mayo de 2004 y mayo de 2005, se incorporaron 14 (CNAP, 2007, p. 38).

Hasta el cese de sus funciones, en 2007, la CNAP había acreditado 55 instituciones ${ }^{9}$ y 379 programas de pregrado (CNAP, 2007, pp. 43-44). La CONAP, en tanto, había evaluado 101 programas de doctorado y 153 de magíster (CNAP, 2007, p. 26).

La aprobación de la nueva Ley de Educación Superior, en mayo de 2018, como se mencionó, puso fin al modelo de acreditación que había consolidado la continuadora de la CNAP y la CONAP. Al llegar a ese año se habían realizado en total 202 procesos de nivel institucional, 2.783 de carreras de pregrado y 1.731 de posgrado ${ }^{10}$.

Estas cifras reflejan el enorme alcance que ha tenido en Chile el sistema de acreditación, lo mismo que los significativos esfuerzos que muchas instituciones de educación superior han hecho por dar fe pública de su calidad.

\section{El aseguramiento de la calidad: origen, implementación e impacto}

La literatura sobre aseguramiento de la calidad tiene ya larga data y ha abarcado una gran variedad de dimensiones. Diversos trabajos se

\footnotetext{
9 Las 55 instituciones se distribuían de la siguiente forma: 22 universidades del Consejo de Rectores, 16 universidades privadas autónomas, nueve institutos profesionales, siete centros de formación técnica y una institución de las fuerzas armadas.

10 Véase www.cnachile.cl
} 
han centrado en analizar las condiciones históricas y contextuales en las que esta idea se expandió a los sistemas de educación superior. Estos sitúan dicho momento en la década de 1980, cuando los gobiernos europeos comenzaron a entregar mayor autonomía a las universidades, pero a cambio exigieron una rendición de cuentas de sus actividades y de la calidad de las mismas (Brockerhoff, 2015).

La relación entre las instituciones y el Estado habría pasado, entonces, de un "modo histórico", basado en regulaciones normativas que definían a priori las actividades posibles de realizar, a otro en que se verificaban ex post los resultados respecto de su consistencia y calidad.

La nueva relación que se ha establecido entre el Estado y las instituciones de educación superior tiene su origen en lo que se ha denominado el "Estado evaluador" o "supervisor" (Brunner, 1990, 1992; Neave, 1998), en las tendencias administrativas asociadas a la Nueva Gestión Pública (Schimank, 2005; Lange \& Schimank, 2007; Bleiklie et al., 2011) y en el establecimiento global de estándares en diversas áreas de la vida social y económica (Brunsson \& Jacobsson, 2002).

Este cambio en el vínculo histórico, además, no solo habría estado motivado por la intención de adecuar la educación superior a las nuevas demandas sociales, sino también por el propósito de preparar a los sistemas estatales para los impactos de la globalización de la educación superior (Vlk, 2006). Un rol destacado lo han jugado diversas organizaciones supranacionales, como UNESCO, la OCDE, el Banco Mundial y el Banco Interamericano de Desarrollo (Medina 2011). Este proceso, sin embargo, no ha estado exento de críticas, en la medida en que la mayor autonomía concedida por los Estados no ha ido acompañada de una desburocratización, sino al contrario (Liuhanen, 2008; Neave, 1998; Newton, 2002).

Los análisis acerca del origen de los procesos de aseguramiento de la calidad también han incluido perspectivas más políticas, que los han visto como una disputa de poder, en cuyo discurso se reflejan sus componentes ideológicos y los grupos en conflicto (Filippakou, 2011). 
En su conjunto, estos estudios dan cuenta de un momento histórico, en el cual la educación superior - y en particular la institución universitaria - ha ido adquiriendo un rol central en las sociedades contemporáneas (Meyer \& Rowan, 2006) y, por lo mismo, estarían surgiendo nuevos mecanismos mediante los cuales ciertos valores sociales y económicos externos han ido adquiriendo mayor peso en la vida institucional de la academia (Brennan \& Shah, 2000).

Junto a este tipo de literatura se ha desarrollado un número significativo de investigaciones e informes nacionales, así como de estudios y simposios que realizan análisis comparados. Estos abordan el tema desde una perspectiva más técnica, referida a las características e implementación de los sistemas de aseguramiento de la calidad en los distintos países o continentes (entre otros, Di Nauta et al., 2004; Bernhard, 2011, NIAD-UE, 2012; Schwarz \& Westerheijden, 2007; OECD, 2013; Lemaitre y Zenteno, 2012).

Un aspecto especialmente relevante ha sido el esfuerzo por precisar el propio concepto de "calidad". Desde los trabajos pioneros de Harvey y Green (1993), que vincularon la calidad al ámbito de la educación superior y formularon los criterios para su conceptualización, el abordaje de este cometido, a juicio de Scharager (2017, p. 23), se puede agrupar en tres tipos de enfoques: "aquellos en los cuales la conceptualización se hace tomando una perspectiva desde dentro de las instituciones de educación superior; aquellos que la definen desde una posición externa, o una combinación de ambas".

En el primero de los casos, el foco está puesto en los procesos internos, en su grado de eficiencia y logro. El segundo se orienta por criterios externos, en los que la rendición de cuentas, que valida dicha calidad, asume la forma de una acreditación. El tercero corresponde a una fusión de ambas perspectivas. A partir de estos enfoques, es posible apreciar el desplazamiento del concepto de "calidad" en un "continuo que va desde aquellas que resaltan la regulación de la calidad a partir de las propias instituciones, hasta aquellas que la asumen como un producto exportable, elaborado en base a exigencias externas" estandarizadas y, por lo mismo, de validez general, más allá de las fronteras nacionales donde se aplican (Scharager, 2017, p. 23). 
Una de las áreas de investigación que ha revelado mayores dificultades metodológicas para alcanzar resultados conclusivos, es la referida al intento de medir el impacto o los efectos de las políticas de aseguramiento de la calidad (Leiber, 2016; Stensaker, 2008; Zapata y Tejeda 2009, p. 203). Los estudios han avanzado en demostrar que éstas han llevado a cambios en las estructuras de gobernanza de las instituciones — fortaleciendo la posición de las administraciones centrales y el poder de los "gestores"y a la formalización de un conjunto de procedimientos internos. Sin embargo, el solo hecho de que estos aspectos hayan sido modificados no es una evidencia de un mejoramiento de la calidad. Más deficitaria aún resulta esta aproximación en áreas como los resultados de la docencia (Banta, 2010). La propia literatura no duda en reconocer esta debilidad y en atribuirla al alto grado de complejidad de la organización universitaria, que no permite identificar qué es "exactamente lo que causó los cambios" (Beerkens, 2018, p. 274), o a la dificultad para determinar causalidad en las intervenciones sociales (Leiber, 2016).

Muchos de los trabajos que han intentado establecer un vínculo entre el aseguramiento de la calidad y ciertos "impactos", tienen una base empírica asociada a entrevistas o encuestas que miden la percepción de los actores, pero no logran evidenciar relaciones directas entre las políticas de aseguramiento de la calidad y desempeños institucionales (Liu et al., 2015; Basantes-Avalos et al., 2016). Otras investigaciones utilizan algunos indicadores indirectos para verificar mejoramientos en las operaciones organizacionales, pero tampoco entregan una perspectiva global de la calidad educativa o institucional (Cinda, 2007; Corengia, 2010; Brenan, 2000; Haapakorpi, 2011; Martínez et al., 2017; Scharager, 2010). Por último, algunos se han centrado en consecuencias administrativas secundarias que las han predicado al aseguramiento de la calidad, pero que no dan cuenta de mejoras de carácter sustantivo (Liston, 2005).

En el extremo, algunos estudios enfocados en Latinoamérica han prescindido incluso completamente de presentar una base empírica en las que sustentar sus afirmaciones, aun cuando éstas se encuentran fundamentadas en el sentido común o en la experiencia 
personal de los investigadores. Estos han remitido los impactos a conceptos genéricos, como el desarrollo de una "cultura de la calidad", de una "autonomía responsable" o de la profesionalización de la gestión (González, 2005). Lo mismo vale para otros trabajos que han intentado aproximaciones basadas en los propios juicios de los acuerdos de acreditación (Atria, 2006, p. 79).

Según algunos autores, "pese a todas estas acciones e interés mostrado por representantes tanto del mundo académico como de la política pública, la evidencia acerca del impacto de estos mecanismos de aseguramiento de la calidad es aún muy escasa" (Scharager 2010, p. 17), y los datos provistos corresponden en general a estudios muy acotados, sino a casos únicos.

Los principales resultados — ya mencionados — apuntan a cambios culturales en las instituciones y a la profesionalización de ciertas áreas de la gestión (Zapata y Tejeda, 2009, p. 202).

Este último punto parece ser el único en el que existe un consenso entre los investigadores, en particular en lo referido a la profesionalización del área de aseguramiento de la calidad al interior de las universidades. Ello refiere tanto a la formalización de procedimientos, como a la formación de equipos que los llevan adelante (Stensaker, 2008).

Ya a mediados de la década del 2000 la literatura identificaba también para el caso de Chile "un desarrollo apreciable de recursos humanos calificados en procesos de evaluación de la calidad" (Lemaitre 2005, p. 64), situación que es refrendada por la literatura reciente (Scharager, 2017).

Este hecho, sin embargo, es juzgado de manera controversial. Por un lado, se ve como algo positivo, al formalizar procedimientos e instalarlos en las universidades, pero, por otro, es considerado de forma negativa, producto de los crecientes y onerosos procesos de burocratización (Beerkens, 2018; Buendía, 2012, Martínez et al., 2017). 
Más allá de las complejidades metodológicas descritas, es un hecho que la gran mayoría de las universidades chilenas ha desarrollado importantes capacidades organizacionales en el área, entendiendo por ello la instalación de rutinas que orientan la toma de decisiones y la obtención de resultados significativos, a partir de asignaciones y combinaciones específicas de recursos (Dávila, 2013; Winter 2003). Estas capacidades organizacionales les han permitido responder de manera satisfactoria a las nuevas demandas planteadas por la acreditación de la calidad. Al menos ello se desprende del volumen alcanzado por estos procesos y el grado de éxito que exhiben.

\section{Aseguramiento de la calidad y desarrollo de} capacidades organizacionales en las universidades chilenas: la mirada desde los datos de acreditación

El análisis que sigue intenta avanzar en responder la pregunta por la relación que ha existido en las universidades chilenas entre su involucramiento en los procesos de acreditación y el desarrollo de capacidades académicas institucionales.

Dos son las hipótesis centrales. La primera plantea que, mientras más temprano el inicio de los procesos de acreditación institucional y mayor cantidad de años obtenidos y áreas acreditadas en el proceso inicial, mayores logros posteriores, gracias a la generación de un conocimiento experto sobre el tema. La segunda que, mientras más temprano el inicio de los procesos de acreditación de carreras, mayor grado de logro y desarrollo académico institucional en el área.

Con el fin de verificar ambas hipótesis se realizó un análisis de regresión con 52 universidades, para determinar la incidencia de un conjunto de variables relacionadas con el desarrollo de capacidades organizacionales en el grado de éxito en los procesos de acreditación institucional.

Complemento del análisis señalado es el estudio de la evolución experimentada por las estructuras organizacionales en el área de aseguramiento de la calidad. Con este objetivo se revisaron los 
organigramas de un conjunto universidades. Primero, se analizaron los cambios sufridos por dichos organigramas en un lapso de diez años, contrastando un grupo de 37 instituciones para las cuales se disponía de información comparativa entre los años 2008-2009 y 2019. Luego, se caracterizó la situación actual (2019) de las áreas asociadas al aseguramiento de la calidad en las 48 universidades para las que se contaba con un organigrama vigente.

El objetivo de este análisis es aportar una perspectiva cualitativa a los resultados obtenidos en el primer procedimiento y reforzar el planteamiento de hipótesis teóricas que puedan contribuir al estudio de los alcances e impactos organizacionales del aseguramiento de la calidad.

a) Desarrollo de capacidades organizacionales y logro en los procesos de acreditación

Con el propósito de establecer una relación entre el grado de logro en los procesos de acreditación institucional y el desarrollo de capacidades organizacionales, se realizó un análisis de regresión lineal múltiple que, respecto de las hipótesis anotadas, consideró como variables independientes: (1) cantidad de años desde el inicio de los procesos de acreditación institucional, hasta 2019; (2) número de años de acreditación otorgados en el primer proceso de acreditación institucional; (3) número de áreas acreditadas en el primer proceso de acreditación institucional, y (4) años desde el inicio de la acreditación de carreras de pregrado hasta 2019. Las variables dependientes son: (1) años de acreditación institucional vigentes en $2018^{11}$; (2) total de áreas acreditadas en el último proceso, ponderadas según complejidad, y (3) porcentaje de programas de pregrado acreditados al 2018. A ellas se sumó, como una cuarta variable dependiente, (4) un índice de desarrollo académico institucional al 2018, que se construyó combinando las variables anteriores. Este índice integra años de acreditación, áreas acreditadas ponderadas de acuerdo a complejidad, y porcentaje de carreras acreditadas, todo

11 Se seleccionó dicho año con el fin de hacer comparable la información, ya que la ley aprobada en mayo de 2018 puso fin a la acreditación de carreras no obligatorias por ley. 
esto de acuerdo al último proceso de acreditación al que se haya sometido la institución ${ }^{12}$. Como variable de control se incluyó el tipo de universidad según régimen de propiedad y adscripción al Consejo de Rectores ${ }^{13}$; los diferentes tipos fueron agregados como variables dummy, dejando fuera, como nivel de comparación, a las universidades privadas nuevas.

La información proviene de diversas fuentes documentales, bases de datos y páginas web. Los datos respecto del primer proceso de acreditación institucional y sus resultados (años y áreas), el año en que se acreditó la primera carrera, así como el número y proporción de programas acreditados por universidades hasta 2018, fueron obtenidos del sitio web de la Comisión Nacional de Acreditación (CNA) y del Sistema de Información de Educación Superior (SIES). Los organigramas correspondientes a 2008 y 2009 se consiguieron a partir de la revisión de material de archivo originado en una investigación anterior y a partir de solicitudes directas a las instituciones. Los correspondientes a 2019 se obtuvieron de la revisión de páginas web, informes de autoevaluación y planes estratégicos.

En la tabla 1 se anotan las características básicas de las variables empleadas.

12 Para la ponderación, se consideró como de mayor complejidad relativa al área de investigación y de menor a la docencia de pregrado, con las áreas de posgrado en lugar intermedio.

13 En el análisis no se consideraron las universidades estatales que comenzaron a funcionar en 2017. Tampoco se consideraron entre las universidades pertenecientes al Consejo de Rectores aquellas que adscribieron a él en el año 2018, con el objeto de no alterar la comparabilidad de los datos. 
Tabla 1.

Estadísticas descriptivas

\begin{tabular}{|c|c|c|c|c|}
\hline Variable & Mínimo & Máximo & Media & Desviación típica \\
\hline $\begin{array}{l}\text { Años desde inicio acreditación } \\
\text { institucional }\end{array}$ & 2 & 15 & 13,29 & 2,033 \\
\hline $\begin{array}{l}\text { Número de años acreditación } \\
\text { otorgados en primer proceso } \\
\text { acreditación institucional }\end{array}$ & 0 & 7 & 2,79 & 1,933 \\
\hline $\begin{array}{l}\text { Número áreas acreditadas en } \\
\text { primer proceso acreditación } \\
\text { institucional }\end{array}$ & 0 & 6 & 2,27 & 1,624 \\
\hline $\begin{array}{l}\text { Número años desde inicio } \\
\text { acreditación carreras pregrado } \\
\text { hasta } 2018\end{array}$ & 0 & 19 & 12,42 & 3,936 \\
\hline $\begin{array}{l}\text { Total de carreras pregrado } \\
\text { acreditadas al } 2018\end{array}$ & 0 & 238 & 35,24 & 39,618 \\
\hline $\begin{array}{l}\text { Porcentaje programas pregrado } \\
\text { acreditados al } 2018\end{array}$ & 0 & 96,5 & 47,648 & 26,143 \\
\hline $\begin{array}{l}\text { Años acreditación vigentes } \\
\text { (último proceso) }\end{array}$ & 0 & 7 & 3,84 & 2,025 \\
\hline $\begin{array}{l}\text { Acreditación en gestión } \\
\text { institucional }\end{array}$ & 0 & 1 & 0,84 & 0,371 \\
\hline $\begin{array}{l}\text { Acreditación en docencia de } \\
\text { pregrado }\end{array}$ & 0 & 1 & 0,84 & 0,371 \\
\hline $\begin{array}{l}\text { Acreditación en vinculación con } \\
\text { medio (en último proceso) }\end{array}$ & 0 & 1 & 0,71 & 0,456 \\
\hline $\begin{array}{l}\text { Acreditación en docencia de } \\
\text { posgrado }\end{array}$ & 0 & 1 & 0,30 & 0,464 \\
\hline Acreditación en investigación & 0 & 1 & 0,45 & 0,502 \\
\hline U. Estatal tradicional & 0 & 1 & 0,03 & 0,183 \\
\hline U. Estatal derivada & 0 & 1 & 0,24 & 0,429 \\
\hline U. Privada tradicional & 0 & 1 & 0,10 & 0,305 \\
\hline U. Privada derivada CRUCH & 0 & 1 & 0,05 & 0,222 \\
\hline $\begin{array}{l}\text { Total de áreas acreditadas último } \\
\text { proceso, ponderada }\end{array}$ & 0 & 11 & 5,80 & 4,158 \\
\hline $\begin{array}{l}\text { Índice agregado de desarrollo } \\
\text { académico institucional }\end{array}$ & 0 & 26,8 & 15,126 & 7,323 \\
\hline $\begin{array}{l}\text { Ubicación de aseguramiento } \\
\text { calidad en Vicerrectoría }\end{array}$ & 0 & 1 & 0,22 & 0,417 \\
\hline $\begin{array}{l}\text { Ubicación de aseguramiento } \\
\text { calidad en staff Rectoría }\end{array}$ & 0 & 1 & 0,50 & 0,506 \\
\hline $\begin{array}{l}\text { Ubicación de aseguramiento de } \\
\text { calidad en unidad dependiente de } \\
\text { Vicerrectoría }\end{array}$ & 0 & 1 & 0,20 & 0,401 \\
\hline $\begin{array}{l}\text { Ubicación de aseguramiento } \\
\text { calidad en otra ubicación }\end{array}$ & 0 & 1 & 0,09 & 0,285 \\
\hline
\end{tabular}

Fuente: elaboración propia. 
Se analizó la incidencia de las variables independientes y de control respecto de cada una de las variables dependientes y al índice agregado. Se generaron así cuatro modelos (M1, M3, M5 y M7). Introduciendo las variables de control se generaron cuatro modelos adicionales (M2, M4, M6 y M8). Todos ellos se registran en la tabla 2. 


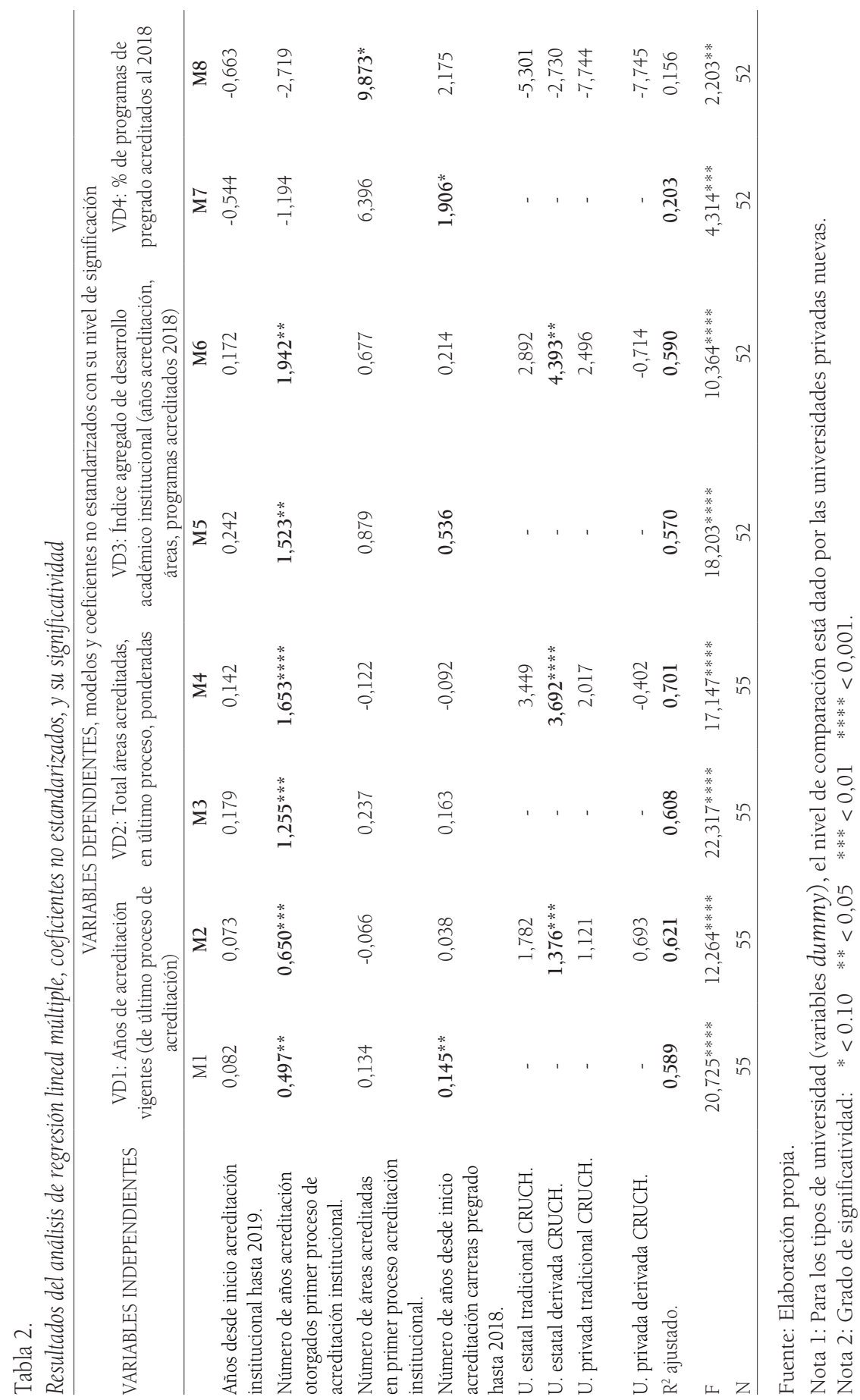


Respecto de la hipótesis 1 , los resultados muestran que el éxito inicial tiene efectos estadísticamente significativos en resultados posteriores a nivel institucional, aunque no cuando se analiza a nivel de programas de pregrado. El tiempo transcurrido, en cambio, no incide por sí solo. Los seis primeros modelos explican una proporción importante de la variación de las variables dependientes, las cuales están referidas al desarrollo institucional (entre 57\% y 70\% de su variación). En los dos últimos modelos, la variable dependiente está específicamente referida al desarrollo de los programas de pregrado; en estos dos casos, la capacidad explicativa del modelo es menor (entre $15 \%$ y $20 \%$ ). Todos los modelos son estadísticamente significativos. Por su parte, las áreas acreditadas al inicio no tienen efectos significativos, salvo respecto del porcentaje de programas de pregrado acreditados en 2018.

En cuanto a la segunda hipótesis, se constata el efecto predicho, del inicio más temprano de los procesos de acreditación de carreras sobre los resultados futuros de esta clase de acreditación. Este efecto, sin embargo, pierde significatividad estadística al controlar por tipo de universidad.

b) El área de aseguramiento de la calidad en la estructura organizacional

Entre fines de 2008 y comienzos de 2009 se recopiló un conjunto de 27 organigramas de distintas universidades chilenas, desde sus páginas web y diversos documentos oficiales (memorias, informes de autoevaluación y planificaciones estratégicas). Durante el segundo semestre de 2019 dicho número fue ampliado a 37, luego de enviar solicitudes a las instituciones restantes. Con propósitos comparativos, se realizó el mismo procedimiento para 2019, lográndose la recopilación de 48 organigramas vigentes.

En los organigramas se identificó, primero, la repartición a cargo del aseguramiento de la calidad. Clave para definir qué unidad seleccionar fue que ésta contuviera, en su nombre oficial, los conceptos "calidad", "evaluación", "autoevaluación" o "acreditación", o que se contara con información oficial que verificara la responsabilidad 
administrativa directa sobre dicha función. Luego de identificada, se determinó el nivel jerárquico en que ésta se encontraba, categorizándose en cuatro posibles: como Vicerrectoría ${ }^{14}(1)$, como staff de Rectoría (2) ${ }^{15}$, como unidad dependiente de Vicerrectoría (3) y otra dependencia (4).

A partir de los datos recopilados se realizaron dos análisis. Primero, un ejercicio que consideraba 37 instituciones con información comparable en un lapso de diez años: 2008/2009 y 2019. En seguida, un análisis descriptivo general del área de calidad de las 48 universidades para las cuales se contaba con organigramas vigentes a 2019.

En la tabla 3 se presenta la ubicación jerárquica de las unidades de calidad entre los años 2008-2009 y 2019.

Tabla 3.

Nivel jerárquico de las unidades de aseguramiento de la calidad, 2008/2009-2019

\begin{tabular}{|c|c|c|c|c|c|c|c|c|c|c|}
\hline \multirow{3}{*}{ Universidad } & \multicolumn{9}{|c|}{ Nivel Jerárquico } & \multirow[t]{3}{*}{ Total } \\
\hline & \multicolumn{2}{|c|}{ Vicerrectoría } & \multicolumn{2}{|c|}{$\begin{array}{l}\text { Staff de } \\
\text { Rectoría }\end{array}$} & \multicolumn{2}{|c|}{$\begin{array}{c}\text { Unidad de } \\
\text { Vicerrectoría }\end{array}$} & \multicolumn{2}{|c|}{ Otro } & \multirow{2}{*}{$\begin{array}{c}\frac{\text { Sin unidad }}{\text { de calidad }} \\
20082009\end{array}$} & \\
\hline & $\begin{array}{l}2008 \\
2009\end{array}$ & 2019 & $\begin{array}{l}2008 \\
2009\end{array}$ & 2019 & $\begin{array}{l}2008 \\
2009\end{array}$ & 2019 & $\begin{array}{l}2008 \\
2009\end{array}$ & 2019 & & \\
\hline Estatal & 1 & 0 & 6 & 9 & 3 & 3 & 3 & 1 & 0 & 13 \\
\hline $\begin{array}{l}\text { Privada } \\
\text { CRUCH }\end{array}$ & 0 & 0 & 3 & 3 & 1 & 2 & 4 & 3 & 0 & 8 \\
\hline Privada & 0 & 8 & 9 & 7 & 4 & 1 & 2 & 0 & 1 & 16 \\
\hline Total & 1 & 8 & 18 & 18 & 8 & 6 & 9 & 5 & 1 & 37 \\
\hline
\end{tabular}

Fuente: elaboración propia.

Entre los años 2008-2009 y 2019, el nivel jerárquico de las unidades de calidad ha evolucionado de manera importante, presentando una interesante diferencia entre los tres tipos de instituciones. Mientras en el sector estatal estas unidades han tendido a ubicarse como staff de Rectoría $(69,2 \%)$ o como una unidad de Vicerrectoría $(23,8)$, en las universidades privadas del CRUCH

14 Dependiente, en consecuencia, del nivel jerárquico más alto: la Rectoría.

15 Para la definición del staff de Rectoría se consideró como un solo nivel a la Rectoría, la Prorrectoría y la Secretaría General. 
presentan una cierta homogeneidad en tres niveles (staff de Rectoría $37,5 \%$, unidad de Vicerrectoría 25\% y otra ubicación 37,5\%), y en el sector privado no perteneciente al Consejo de Rectores se ha producido un movimiento ascendente en la jerarquía de la función de calidad.

En este sentido, dos situaciones resultan llamativas en los diez años que cubre este análisis. Primero, el hecho que hacia 20082009 solo una universidad (estatal) del Consejo de Rectores tenía una Vicerrectoría a cargo del tema de calidad. Esta función fue luego subsumida en otra unidad de igual rango, pero que ya no consideraba el concepto "calidad" en el nombre de la repartición principal, sino que lo trasladó a la unidad secundaria a cargo.

La otra situación interesante es la ocurrida en el sector privado no perteneciente al Consejo de Rectores. Mientras en los años 2008-2009 ninguna de las 16 universidades para las que se logró recopilar información desarrollaba esta función en el nivel de Vicerrectoría, al llegar a 2019 ocho de ellas le habían asignado dicha jerarquía (llegando a 10, si se considera aquellas para las que no había información comparable). De igual modo, se produjo un descenso de aquellas que se ubicaron como staff de Rectoría (de 9 a 7), lo mismo que aquellas que dependían de una Vicerrectoría (de 4 a 1) o de otra instancia de menor rango (de 2 a 0). Esta evolución sin duda está relacionada con la relevancia que el aseguramiento de la calidad fue adquiriendo, especialmente luego de la aprobación de la Ley 20.129, en 2006, y de la vinculación entre resultados en los procesos de acreditación y el acceso a recursos públicos.

Al analizar la situación que presentaban las unidades de calidad en 2019, en las 48 instituciones para las que se dispuso de un organigrama oficial, se obtienen los resultados presentados en la tabla 4. 
Tabla 4.

Nivel jerárquico de las unidades de aseguramiento de la calidad, 2019

\begin{tabular}{lccccc}
\hline Universidad & \multicolumn{5}{c}{ Nivel jerárquico 2019 } \\
\cline { 2 - 6 } & Vicerrectoría & $\begin{array}{c}\text { Staff de } \\
\text { Rectoría }\end{array}$ & $\begin{array}{c}\text { Unidad de } \\
\text { Vicerrectoría }\end{array}$ & Otro & Total \\
\hline Estatal & 0 & 11 & 5 & 2 & 18 \\
Privada CRUCH & 0 & 4 & 2 & 3 & 9 \\
Privada & 10 & 9 & 2 & 0 & 21 \\
Total & 10 & 24 & 9 & 5 & 48 \\
\hline
\end{tabular}

Fuente: elaboración propia.

Los resultados del análisis de la totalidad de los organigramas 2019 (tabla 4) tiene un alto nivel de correspondencia con los arrojados por el ejercicio comparativo 2008-2009 y 2019. Mientras las universidades estatales concentran las unidades de calidad en el nivel de staff de Rectoría (61\%) y de dependencia de Vicerrectoría $(27,8 \%)$, las instituciones privadas del CRUCH las distribuyen de manera más homogénea entre staff de Rectoría (44,4\%), unidad de Vicerrectoría $(22,2 \%)$ y otra dependencia (33,3\%). Las universidades privadas no pertenecientes al CRUCH, en tanto, las concentran en el nivel de Vicerrectoría $(47,6 \%)$ y staff de Rectoría $(42,9 \%$ ).

Otra perspectiva interesante de análisis es revisar el vínculo que las unidades de calidad tienen con otras áreas de la universidad, ya sea porque estas unidades cumplen más de una función o porque se encuentran subordinadas a una instancia que atiende, además, otras tareas (tabla 5).

Tabla 5.

Vinculo de las unidades de calidad con otras áreas, 2019

\begin{tabular}{lccccc}
\hline Universidad & $\begin{array}{c}\text { Unidades de Solo calidad } \\
\text { calidad }\end{array}$ & $\begin{array}{c}\text { Área } \\
\text { académica }\end{array}$ & Planificación & Análisis \\
\hline Estatal & 18 & 9 & 3 & 6 & 2 \\
Privada CRUCH & 9 & 3 & 2 & 3 & 2 \\
Privada no CRUCH & 21 & 8 & 1 & 8 & 5 \\
Total & 48 & 20 & 6 & 17 & 9 \\
\hline
\end{tabular}

Fuente: Elaboración propia.

A partir de este análisis se constata que no existen diferencias significativas en la manera en que los tres tipos de instituciones 
vinculan las unidades de calidad con otras funciones institucionales. Una mención merece el hecho que el 50\% de las unidades de calidad del sector estatal tiene solo a cargo dicha función. En general, sin embargo, aparecen vinculadas al área académica (como dependencia de tal Vicerrectoría, por ejemplo), a planificación estratégica (y todos sus derivados: gestión estratégica, desarrollo, etc.) o a análisis institucional y estudios. Esta combinación de funciones es también válida para las diez vicerrectorías de aseguramiento de la calidad identificadas en las universidades privadas no pertenecientes al $\mathrm{CRUCH}$, que solo en cuatro ocasiones están referidas únicamente a esa función.

Para analizar el efecto diferencial que pudiera tener una u otra ubicación de las unidades de aseguramiento de la calidad, llevamos a cabo un análisis de regresión lineal múltiple, considerando como variable dependiente el índice agregado de desarrollo académico institucional. Los resultados se muestran en la tabla 6.

Tabla 6 .

Modelos de análisis de regresión múltiple, coeficientes no estandarizados y su significatividad

VD3: Índice agregado de desarrollo académico institucional (años acredit. áreas, programas acreditados 2018)

\begin{tabular}{lcc} 
& Ml & M2 \\
\hline $\begin{array}{l}\text { Ubicación de aseguramiento de } \\
\text { calidad en Vicerrectoría }\end{array}$ & $-6,159^{* *}$ & $-1,973$ \\
Ubicación en staff de Rectoría & $-4,667^{* *}$ & $-2,963$ \\
Otra ubicación & 1,037 & $-0,037$ \\
U. estatal tradicional CRUCH & & $9,301^{* *}$ \\
U. estatal derivada CRUCH & & 2,870 \\
U. privada tradicional CRUCH & & $8,446^{* * *}$ \\
U. privada derivada CRUCH & & 1,279 \\
& 0,127 & \\
$R^{2}$ ajustado & $3,129^{* *}$ & 0,272 \\
F & 44 & $3,353^{* * *}$ \\
$\mathrm{~N}$ & & 44 \\
\hline
\end{tabular}

Fuente: Elaboración propia.

Nota 1: Para los tipos de universidad (variables dummy), el nivel de comparación está dado por las universidades privadas nuevas.

Nota 2: Grado de significatividad: $\quad *<0.10 \quad * *<0,05 \quad * * *<0,01 \quad * * * *<0,001$

Nota 3: Para las variables sobre ubicación del aseguramiento de la calidad, el nivel de referencia es unidad dependiente de Vicerrectoría. Para tipo de universidad, es privada nueva. 
De acuerdo con los resultados de este análisis, se puede apreciar que el nivel de ubicación en la jerarquía institucional nivel de Vicerrectoría - juega un rol importante en los resultados obtenidos en los procesos de acreditación. No obstante, al controlar dicha variable por tipo de universidad esa diferencia se hace no significativa.

\section{Conclusión}

Como ha sido posible apreciar en las páginas precedentes, a veinte años de iniciados los procesos de acreditación, el sistema de educación superior chileno los ha emprendido y desarrollado con gran intensidad. Ello se expresa no solo en la fecunda producción de normativa e institucionalidad, sino también en la activa participación de las universidades en dichos procesos y en las modificaciones que éstas han hecho a sus estructuras organizacionales.

En la totalidad de las instituciones para las cuales fue posible obtener un organigrama (48), se aprecia que éstas disponen de una unidad dedicada al aseguramiento de la calidad, ya sea en forma exclusiva o vinculada a otras áreas, como planificación estratégica o análisis institucional. Este hecho corresponde, cuando menos formalmente, al desarrollo de capacidades organizacionales que, con seguridad, han favorecido los resultados en los procesos de acreditación emprendidos.

Los análisis llevados a cabo muestran que existe una relación entre la fecha de inicio de los procesos de acreditación (tanto a nivel institucional como de programas) y los logros en ellos (medidos como años de acreditación otorgados y número de áreas acreditadas), con los resultados posteriores en la acreditación institucional. Lo anterior no se expresa de igual forma en el ámbito de las carreras y programas.

Una causa de este resultado puede estar referida a los diferentes grados de centralización y alcances de estos procesos. Mientras en el nivel institucional ellos se realizan desde una unidad central y dependen en su mayor parte de la capacidad de gestión de la administración, en el nivel de carreras y programas dependen, en 
una proporción importante, tanto en sus contenidos como en su gestión, de las capacidades locales de las unidades académicas. Éstas, como es sabido, pueden presentar diferencias significativas al interior de una misma institución.

El mayor grado de logro exhibido evidencia que, más allá de las condiciones iniciales en que se encontraba la institución, ha existido un desarrollo de capacidades organizacionales para enfrentar estos procesos, que se expresa en la relación de las variables independientes con el índice agregado de desarrollo académico institucional.

Reflejo de ello es, además, el hecho de que todas las instituciones cuyo organigrama fue analizado contaban con unidades de aseguramiento de la calidad.

Dichas unidades, no obstante, exhiben una particularidad en la evolución que han tenido en la estructura jerárquica en los últimos diez años, que se correlaciona con la forma en que característicamente se han agrupado las universidades chilenas. Aquellas instituciones que conforman el Consejo de Rectores han tendido a situarlas en el nivel de staff de Rectoría o como una dependencia de Vicerrectoría. Las universidades privadas, fundadas en la década de 1980, en tanto, las ubican como staff de Rectoría o les asignan el estatus de vicerrectorías. Así, mientras en el Consejo de Rectores se eliminó la única Vicerrectoría de Calidad que existía hacia 2008-2009, en el sector privado no perteneciente a él se cuenta actualmente con diez unidades de tal jerarquía.

Respecto de este llamativo fenómeno es posible plantear dos hipótesis. La primera se refiere al eventual desarrollo de procesos de isomorfismo organizacional al interior de grupos diferenciados de instituciones. La teoría neoinstitucional ha hecho un esfuerzo por mostrar cómo ciertas normas, valores y hábitos condicionan la acción de las organizaciones y las impulsan a emular instituciones ya establecidas (Dimaggio \& Powell, 1983; Meyer \& Rowan, 1977; Zucker, 1977, 1988). Estas presiones homogeneizadoras provienen tanto del entorno institucional de las organizaciones como de su propio interior y se expresan de tres maneras: como 
isomorfismo coercitivo, mimético y normativo. En el primero su origen se encuentra fuera de la organización y, en especial, en el Estado, que provoca fuertes tendencias homogeneizadoras (mediante marcos legales comunes, procedimientos estandarizados o en tanto principal fuente de financiamiento). El segundo (isomorfismo mimético) se produce debido a que las organizaciones tienden a copiar a aquellas que perciben como más exitosas. Por último, el isomorfismo normativo se debe a que los profesionales contratados por las organizaciones han sido socializados en normas dominantes e intentan replicar lo que ya conocen.

Desde esta perspectiva, entonces, es posible plantear la hipótesis de que la evolución organizacional diferenciada que han sufrido las unidades de calidad entre las instituciones del Consejo de Rectores y aquellas no pertenecientes a él, puede tener su origen en procesos isomórficos derivados de los conglomerados a los que se integran. Mientras el sector estatal y privado tradicional (y derivado) han conformado históricamente el Consejo de Rectores, las universidades privadas fundadas con posterioridad a 1980 no disponen de una orgánica única. La existente — la Corporación de Universidades Privadas, CUP - aglutina solo a 11 instituciones (sobre 29 vigentes en 2019), de las cuales seis exhiben este tipo de desarrollo. Estas coinciden, además, con aquellas acreditadas institucionalmente. Una séptima, también acreditada, no da a la unidad de aseguramiento de la calidad ese rango institucional. De las restantes cuatro instituciones, tres no se encuentran acreditadas ${ }^{16}$. A ello se agrega el hecho de que algunas universidades privadas (3) son filiales de consorcios internacionales y, por lo mismo, es probable que sigan modelos organizacionales similares. Como sea, al establecerse vínculos más estrechos entre distintos grupos de organizaciones, es probable que tengan lugar importantes procesos de isomorfismo normativo o mimético que hayan llevado a la diferenciación descrita.

Una segunda hipótesis puede atribuir la instalación de las unidades de calidad en el más alto nivel jerárquico de la institución (Vicerrectoría) a una estrategia de "señalización de mercado". La

16 Corporación de Universidades Privadas. https://www.cupchile.cl 
teoría de la señalización (signaling) explica cómo algunas acciones altamente costosas de las personas o las organizaciones pueden justificarse como una forma de señalizar al mercado una superioridad en un desempeño, que hace relativamente menos costosa esa acción para esa persona u organización que para otros competidores ${ }^{17}$.

En el ámbito de la educación superior, este fenómeno ha sido extensamente estudiado, en especial en el sistema de educación superior norteamericano. La señalización de mercado en este contexto se expresa, en general, en la contratación de "estrellas" académicas, en la alta prioridad que se asigna a la publicidad y las relaciones públicas, en políticas de becas notoriamente visibilizadas, en obtención de certificaciones de acreditación y en la participación en rankings (Mause, 2009, p.1120; Newman, Couturier \& Scurry, 2004, pp. 14-15). Para el mismo caso norteamericano, Siow (1997) evidencia que la investigación funciona como señalización de calidad para los estudiantes de más alta aptitud académica, aun cuando no para el grueso de ellos. En este contexto, entonces, es posible plantear la hipótesis de que la instalación de unidades de calidad en el nivel de Vicerrectoría podría tener relación con el intento de señalizar al entorno un estándar de calidad superior, ya sea para el público general, hacia otras instituciones similares o para las instancias públicas a cargo de su control.

Esta hipótesis se ve refrendada en él último análisis de regresión realizado (tabla 6), cuyos resultados permiten plantear que instituciones tradicionales más consolidadas no requieren de efectos de señalización para representar calidad académica. Por esta misma razón, las unidades asociadas al aseguramiento de la calidad se encuentran en niveles jerárquicos inferiores, al contrario de lo que estaría sucediendo con parte del sector privado no perteneciente a él.

Responder estas hipótesis, sin embargo, escapa a las pretensiones de este artículo, pero su enunciación busca abrir otros caminos de exploración para este fenómeno.

17 Para antecedentes de esta teoría, ver a Spence, 1973; Arrow, 1973 y Stiglitz, 1975. 
Lo que sí es posible afirmar es que, tras 20 años de procesos de acreditación, ha habido un importante desarrollo de capacidades organizacionales en las universidades chilenas, lo que ha permitido mayores logros en ellos, generando un círculo, al menos en este sentido, virtuoso.

Si esto se expresa también en impactos efectivos en la calidad de los procesos formativos o en la eficiencia de la gestión de las instituciones, sigue siendo una pregunta abierta, como en la mayoría de la literatura revisada.

\section{Referencias}

Arrow, K. (1973). Higher Education as a Filter. Journal of Public Economics, 2, 193-216.

Atria, R. (2006). La gestión de la universidades del Consejo de Rectores. Calidad en la Educación, 24, 67-116.

Banta, T. W. (2010). Impact of Addressing Accountability Demands in the United States. Quality in Higher Education, 16(2), 181-183

Basantes-Avalos, R., Coronel-Sánchez, J. y Vinueza-Jara, A. (2016). Impacto de la evaluación y acreditación de las carreras profesionales ofertadas por la Universidad de Cimborazo desde la percepción de los estudiantes. Revista de Ciencia UNEMI, 9(21), 36-47.

Beerkens, M. (2018). Evidence-based policy and higher education quality assurance: progress, pitfalls and promise. European Journal of Higher Education, 8(3), 272-287.

Bernasconi, A. y Rojas, F. (2004). Informe sobre la Educación Superior en Chile: 1980-2003. Santiago de Chile: Editorial Universitaria.

Bernhard, A. (ed.) (2011). Quality Assurance in an International Higher Education Area. A Case Study Approach and Comparative Analysis. Wiesbaden, Alemania: VS Verlag für Sozialwissenschaften.

Bleiklie, I., Enders, J., Lepori, B. \& Musselin, C. (2011). New Public Management, Network Governance and the University as a Changing Professional Organization. In T. Christensen y P. Laegreid. The Ashgate Research Companion to New Public Management, (pp. 161-176). Farnham, United Kingdom: Ashgate.

Brennan, J. \& Shah, T. (2000). Quality Assessment and Institutional Change: Experiences from 14 Countries. Higher Education, 40, 331-349. 
Brockerhoff, L., Huisman, J. \& Laufer, M. (2015). Quality in Higher Education: A literature review. Belgium: Centre for Higher Education Governance, Ghent University.

Brunner, J. J. (1990). Estado e instituciones de educación superior en América Latina: tres modelos de relaciones. En C. Cox (Ed.), Formas de gobierno en la educación superior: nuevas perspectivas, (pp. 299-313). Santiago de Chile: Colección Foro de la Educación Superior

Brunner, J. J. (1992). Evaluación y financiamiento de la educación superior en América Latina: bases para un nuevo contrato. Documento de Trabajo. Santiago de Chile: Flacso.

Brunsson, N. \& Jacobsson, B. (2002). A World of Standards. Oxford, England: Oxford University Press.

Buendía, A. (2012). Evaluación y acreditación de programas en México. Más allá de los juegos discursivos. Diálogos sobre Educación, 3(6), 1-19.

CINDA. (2007). Acreditación y Dirección Estratégica para la Calidad de las Universidades. Santiago de Chile: CINDA.

CNAP. (2007). El modelo chileno de acreditación de la educación superior. Santiago de Chile: CNAP-MINEDUC.

Comisión de Estudios de la Educación Superior. (1991). Una política para el desarrollo de la educación superior en la década de los noventa. Santiago de Chile.

Corengia, Á. (2010). Impacto de la política de evaluación y acreditación de la calidad en universidades de la Argentina. Estudios de casos (Tesis doctoral). Universidad de San Andrés, Argentina.

Dávila, J. C. (2013). Capacidades orgnizacionales: dinámicas por naturaleza. Cuadernos de Administración, 26(47), 11-33.

Di Nauta, P., Pirjo-Liisa, O., Schade, Angelika \& Scheele, J. P. (eds.) (2004). Accreditation Models in Higher Education. Experiences and Perspectives. ENQA Workshop Reports 3.

DiMaggio, P. \& Powell, W. (1983). The Iron Cage Revisited: Institutional isomorphism and Collective Rationality in Organizations Fields. American Sociological Review, 48(2), 147-160.

Fernández Darraz, E. (2015). Políticas públicas de educación superior desde 1990 hasta el presente. En A. Bernasconi (Ed.), La educación superior de Chile. Transformación, desarrollo y crisis (pp. 173-217), Santiago de Chile: Ediciones Universidad Católica de Chile. 
Filippakou, O. (2011). The Idea of Quality in Higher Education: A Conceptual Approach. Discourse: Studies in the Cultural Politics of Education, 32(1), 15-28.

Garreta, N. (1998). Hacia el Plan Nacional de Evaluación de las Calidad de las Universidades. Revista de Educación, 315, 283-292.

González, L. (2005). El impacto del proceso de evaluación y acreditación en las universidades de América Latina. En I. Lavados (Ed.), Los procesos de acreditación en el desarrollo de las universidades. Santiago de Chile: CINDA. Recuperado de http://www.luisedogonzalez.cl/ pdf/2005/2005-07.pdf, 20 de mayo 2019.

Haapakorpi, A. (2011). Quality assurance processes in Finnish universities: direct and indirect outcomes and organisational conditions. Quality in Higher Education, 17(1), 69-81.

Harvey, L. \& Green, D. (1993). 'Defining Quality', Assessment \& Evaluation. Higher Education, 18(1), 9-34.

Lange, S. \& Schimank, U. (2007). Zwischen Konvergenz und Pfadabhängigkeit: New Public Management in den Hochschulsystemen fünf ausgewählter OECD-Länder. In K. Holzinger, H. Jörgens, y C. Knill (Eds.), Transfer, Diffusion und Konvergenz von Politiken. (pp. 522-548). Wiesbaden, Germany.

Leiber, T. (2016). Impact Evaluation of Quality Assurance in Higher Education. A Manual. Mannheim, Germany: IMPALA.

Lemaitre, M. J. (2005). Aseguramiento de la calidad en Chile: impacto y proyecciones. En: Seminarios de la Calidad: Impacto y proyecciones (pp. 55-69). Santiago de Chile: Consejo Nacional de Educación.

Lemaitre, M. J. y Zenteno, M. E. (2012). Aseguramiento de Calidad en Iberoamérica. Educación Superior. Informe 2012. Santiago de Chile: Proyecto Alfa-Universia-CINDA.

Liston, C. (2005). El impacto de las auditorías de aseguramiento de la calidad en las asociaciones educacionales transnacionales. En: Seminarios de la Calidad: Impacto y proyecciones (pp. 29-54). Santiago de Chile: Consejo Nacional de Educación.

Liu, S., Tan, M. \& Meng, Z. (2015). Impact of Quality Assurance on Higher Education Institutions: A Literature Review. Higher Education Evaluation and Development, 9(2), 17-34.

Liuhanen, A-M. (2008). How are University Evaluations Used. The Perspectives of two Finnish Universities (Tesis doctoral), University of Tampere, Finlandia. 
Mairata, M. J. (2001). Modelos y sistemas de evaluación de la calidad en la universidad española. Educació i Cultura, 14, 133-146.

Martínez, J., Tobón, S. y Romero, A. (2017). Problemáticas relacionadas con la acreditación de la calidad de la educación superior en América Latina. Innovación Educativa, 17(73), 79-96.

Mause, K. (2009). Too Much Competition in Higher Education? Some Conceptual Remarks on the excessive-signaling Hypothesis. American Journal of Economics and Sociology, 68(5), 1107-1133.

Medina, S. R. (2011). Los organismos internacionales y la evaluación como política educativa en México: elementos para un balance. En: S. R. Medina. Políticas y educación. La construcción de un destino (pp. 17-38). México DF: Ediciones Díaz de Santos/UNAM.

Meyer, H. D. y Rowan, B. (Eds.). (2006). The New Institutionalism in Education. Albany, USA: State University of New York Press.

Meyer, J. W. \& Rowan, B. (1977). Institutionalized Organizations: Formal structures as myth and Ceremony. American Journal of Sociology, 83(2), 340-363.

Neave, G. (1998). The Evaluative State Reconsidered. 20th Anniversary Issue of Review of Trends in Higher Education. European Journal of Education, 33(3), 265-284.

Newman, F., Couturier, L. \& Scurry, J. (2004). The Future of Higher Education: Rhetoric, Reality, and the Risks of the Market. San Francisco, USA: JosseyBass.

Newton, J. (2002). Barriers to Effective Quality Management and Leadership: Case Study of Two Academic Departments. Higher Education, 44(2), 182- 212 .

NIAD-UE. (2012): Quality Assurence for Higher Education in Japan. Tokyo, Japón: NIAD-UE.

OECD. (2013). Quality Assurance in Higher Education in Chile 2013. OECD Publishing.

Presidencia de la República. (1992). Mensaje 392-324. Proyecto de ley que modifica la Ley No 18.962, Orgánica Constitucional de Enseñanza.

Presidencia de la República. (2006). Ley 20.129 que Establece un Sistema Nacional de Aseguramiento de la Calidad de la Educación Superior.

Presidencia de la República. (2018). Ley 21.091, sobre Educación Superior.

Scharager, J. (2010). Impacto de las políticas de aseguramiento de la calidad en programas de educación superior: un estudio exploratorio. Calidad en la Educación, 32, 16-42. 
Scharager, J. (2017). Nuevos actores en un viejo escenario: la profesionalización de la gestión de la calidad académica en Chile, 1990-2015 (Tesis Doctoral). Universidad de Leiden, Holanda.

Schimank, U. (2005). 'New Public Management' and the Academic Profession: Reflections on the German Situation. Minerva, 43, 361-376.

Schwarz, S. \& Westerheijden, D. (Eds.). (2007). Accreditation and Evaluation in the European Higher Education Area. Dordrecht, Holland: Springer.

Siow, A. (1997). Some Evidence on the Signaling Role of Research in Academia. Economics Letters, 54, 271-276.

Spence, M. (1973). Job Market Signaling. Quarterly Journal of Economics, 87 , 355-379.

Stensaker, B. (2008). Outcomes of Quality Assurance: A Discussion of Knowledge, Methodology and Validity. Quality in Higher Education, 14(1), 3-13.

Stiglitz, J. (1975). The theory of Screening, Education, and the Distribution of Income. American Economic Review, 65, 283-300.

Vlk, A. (2006). Higher Education and the GATS: Regulatory Consequences and Stakeholders' Responses (Tesis doctoral). Universidad de Twente, Países Bajos.

Winter. S. G. (2003). Understanding dynamic capabilities. Strategic Management Journal, 24(10), 991-995.

Zapata, G. (2004). Acreditación institucional en Chile: una opción emergente. Calidad en la Educación, 21, 141-154.

Zapata, G. y Tejeda, I. ((2009). Impactos del aseguramiento de la calidad y acreditación de la educación superior. Consideraciones y proposiciones. Calidad en la Educación, 31, 192-209.

Zucker, L. G. (1977). The Role of Institutionalization in Cultural Persistence. American Sociological Review, 42, 726-743.

Zucker, L. G. (1988). Institutional Theories of Organization. American Review of Sociology, 13, 443-464. 
Fuentes de Información

Comisión Nacional de Acreditación, CNA. www.cnachile.cl

Consejo de Rectores de las Universidades Chilenas, CRUCH. www.cruch.cl Corporación de Universidades Privadas, CUP. www.cupchile.cl

Mi Futuro. Subsecretaría de Educación Superior, Ministerio de Educación de Chile. www.mifuturo.cl

Recibido: 09/06/2020

Aceptado: 25/09/2020 